\title{
A Probabilistic Multiscale Approach to Hysteresis in Shear Wave Propagation in Biotissue
}

\author{
H.T. Banks \\ Center for Research in Scientific Computation \\ North Carolina State University \\ Raleigh, NC 27695-8205 \\ Gabriella A. Pinter \\ Department of Mathematical Sciences \\ University of Wisconsin, Milwaukee \\ Milwaukee, WI 53201-0413
}

January 31, 2004

\begin{abstract}
Motivated by a problem involving wave propagation through viscoelastic biotissue, we present a theoretical framework for treating hysteresis as multiscale phenomena which must be averaged across distributions of internal variables. The resulting systems entail probability measure dependent partial differential equations for which we establish well-posedness in a framework that leads readily to computationally useful approximations.
\end{abstract}

Keywords: multiscale modeling, hysteresis, wave propagation, biotissues, probability measures, Prohorov metric

\section{Introduction}

At the heart of modern day sciences (especially the computational sciences) of materials, biology, etc., is the challenge of effectively using the explosion of available information at micro (genomic, atomic, molecular, nano, etc.) levels to develop more and more accurate biologically and physically based system response (macro level) models for use in prediction, control, design, and simple organism/structure simulations. A number of fundamental issues (e.g., how to model uncertainty/variability in heterogeneous materials) in multiscale modeling and control are important in addressing this challenge. In this presentation we discuss 
some of these issues in the context of internal dynamics (molecular level) in system response models (organism level). More precisely, we offer a probabilistic based internal strain variable framework to treat nonlinear hysteresis arising in propagation of shear waves in biotissues. The usual models for such problems involve integro-partial differential equations which are most often phenomenological in nature as well as being computationally challenging. Our approach is founded on the belief that hysteresis is actually a manifestation of the presence of multiple scales in a physical or biological system that is frequently modeled (and masked) with a phenomenological representation such as an hysteresis integral for the macroscopic stress-strain constitutive law ( e.g., for models of viscoelastic damping via hysteretic integrals versus internal variable representations, see [13] and the references therein). The approach we present embodies a multiscale treatment of hysteresis as resulting from a continuum of microscopic or molecular level internal strain mechanisms averaged (with respect to a material dependent probability distribution) across populations of molecules. Such an approach using nonlinear stick-slip molecular reptation models for both tensile and shear deformations has recently [11],[12] been developed and successfully used in computational efforts with composite rubbers. The probabilistic framework we present here has its origins in work on distributed growth rates for marine (mosquitofish) populations in size-structured models as developed in [1],[5],[6],[7],[8]. We develop here for the first time a theoretical framework for molecular based strain models which readily leads to efficient computational methods that offer an alternative to the computationally intensive phenomenological viscoelastic soft tissue kernels proposed by Fung and his colleagues. In [22], Fung lays the foundations for the essentials of polymer biomaterials such as elastin, fibers, collagen, etc., which experimentally manifest classical hysteretic behavior in tension and shear (see p. 261, [22]). He furthermore cogently argues the essential nonlinear nature of these materials. He also explains the need for continuous spectrum models (e.g., a continuum of relaxation parameters ) due to the strain rate independence of the stress-strain law ([22] p. 281-287) over several decades of frequencies (see also [21], [25], [26], [28]). This led to the quasi-linear Fung kernel ( see (2.6) below) in the context of Boltzman type model (2.5) constitutive laws. The formulation we give here also provides continuous spectrum models, although we subsequently report on calculations in [2] with finite spectrum approximate models (Section 6 below). The computational results suggest that such models will often suffice to describe well the type of deformations arising in the wave propagation problems motivating our developments in this paper.

Our motivating problem arose in joint collaborations with scientists and engineers at MedAcoustics, Inc., as part of the Industrial Applied Mathematics Program at N.C. State University ( see http://www.ncsu.edu/crsc/ ). We summarize briefly here the salient points of this problem, while referring the reader to [2] and [14] for more details. Turbulent blood flow due to arterial stenosis in partially occluded arteries produces normal forces on arterial walls. This results in vibrations in the surrounding body tissue which are transmitted to body surfaces in two forms: compressional waves and shear waves. The shear waves are at low frequencies $(\leq 2 \mathrm{kHz})$ with low propagation speed and attenuation. Devices involving multiple arrays of piezoceramic sensors were developed at MedAcoustics, with the goal of measuring shear wave propagation at the surface of the chest. The resulting signals are then 
processed to determine the location and extent of the stenosis. A part of this overall detection and diagnostic problem is the focus of our efforts: modeling of the shear wave propagation through a heterogeneous chest cavity. The cavity is composed of soft body tissues (lung, muscular and connective tissue) as well as bone. Here we consider shear wave propagation through a viscoelastic heterogeneous medium. At MedAcoustics, early experiments and data collection were carried out on cylindrical shaped mold of tissue-like synthetic gel as depicted schematically in Figure 1. The cylinder of gel surrounded a tube in which source disturbances simulating disrupted flows were produced. Thus the tube with disturbances mimicked an artery with stenosis. Shear waves were measured with sensor arrays mounted on the gel outer surface. Subsequent experiments involved data collection on pigs (who, unfortunately for them, have cylindrical-like chest geometries!!). In [2], the authors developed and used a onedimensional (axial symmetry was assumed) homogeneous medium model for computations and analysis. Here we consider an extension of that model which allows for "molecular" level heterogeneity in the tissue (gel), while retaining the overall geometry and axial symmetry. An inner radius $R_{1}$ and outer radius $R_{2}$ for the gel is assumed, with the gel initially at rest. The outer surface of the gel is a free surface.

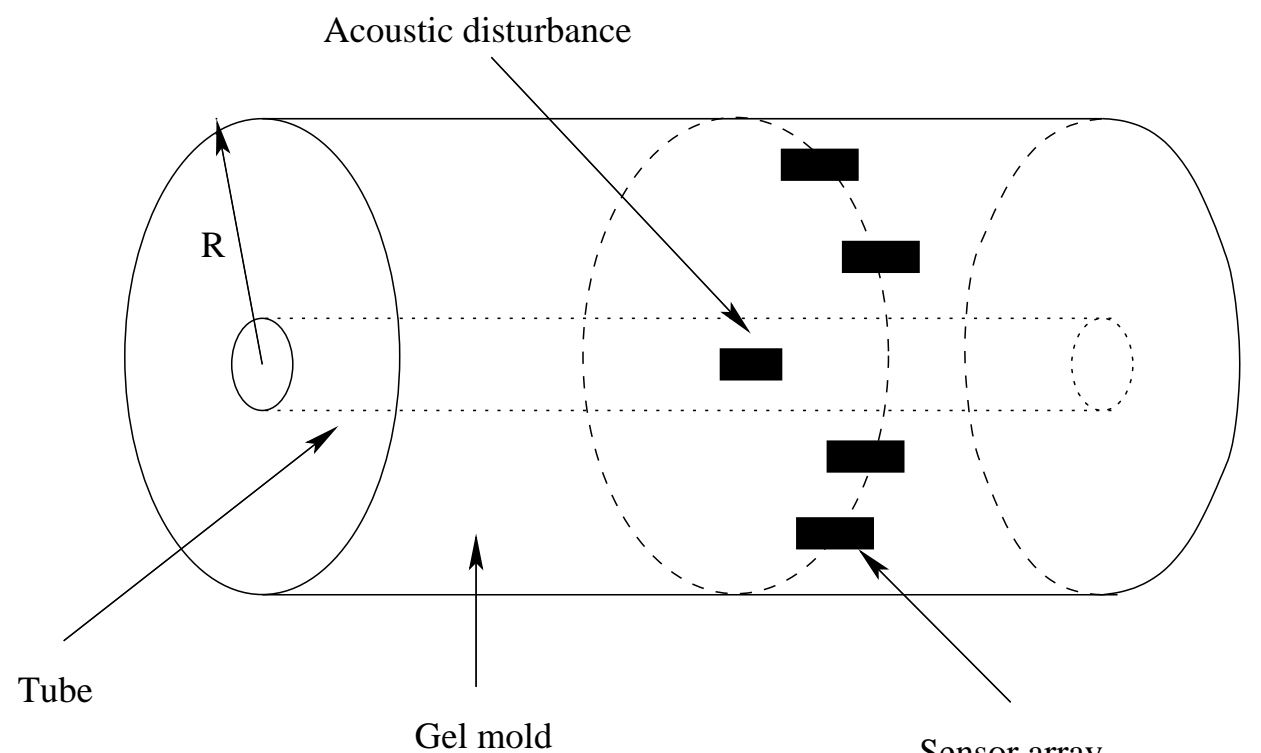

Gel mold

Sensor array

Figure 1: Cylindrical model geometry with artificial "stenosis" and piezo sensors

\section{Problem formulation}

In [2] and [14] the following one-dimensional model (see Figure 1) is introduced to describe the propagation of shear waves in soft tissue

$$
\rho \frac{\partial^{2} u}{\partial t^{2}}-\frac{\partial}{\partial x} \sigma(t, x)=F(t, x), \quad R_{1}<x<R_{2}
$$


where $\rho$ is the mass density, $u$ is the Cartesian shear displacement, $\sigma$ is the shear stress and $F$ represents a body forcing term. We assume that at the inner or left boundary, $\left(x=R_{1}\right)$, we have a pure shear shearing force, while the outer or right boundary, $\left(x=R_{2}\right)$, is a free surface

$$
\sigma\left(t, R_{1}\right)=f(t), \text { and } \sigma\left(t, R_{2}\right)=0
$$

The initial conditions are

$$
u(0, x)=u_{0}(x), \text { and } u_{t}(0, x)=u_{1}(x), \quad R_{1}<x<R_{2}
$$

To complete the model one needs to find an adequate constitutive relationship that relates the shear stress, $\sigma$, and strain, $\varepsilon=u_{x}(t, x)$, in soft tissue (arteries, muscle, skin, lung, etc., ...). In [2] an internal strain variable model is considered in the following form

$$
\sigma(t, x)=C_{D} \dot{\varepsilon}(t, x)+\sum_{j=1}^{N} \varepsilon_{j}(t, x),
$$

with

$$
\begin{aligned}
& \frac{d \varepsilon_{j}(t)}{d t}=-\frac{1}{\tau_{j}} \varepsilon_{j}(t)+C_{j} \frac{d}{d t} \sigma_{e}\left(u_{x}(t)\right) \\
& \varepsilon_{j}(0, x)=0, \quad j=1, \ldots N,
\end{aligned}
$$

where $\sigma_{e}$ is the elastic response function defined in Fung $([22], \S 7)$ and given as

$$
\sigma_{e}\left(u_{x}\right)=\gamma+\beta e^{\alpha u_{x}}
$$

On the other hand, Fung proposes the constitutive relationship

$$
\sigma(t)=\int_{0}^{t} G(t-s) \frac{d \sigma_{e}\left(u_{x}\right)}{d s} d s
$$

where $G(t)$ is the reduced relaxation function given in the form

$$
G(t)=\left\{1+C\left[E_{1}\left(\frac{t}{\tau_{2}}\right)-E_{1}\left(\frac{t}{\tau_{1}}\right)\right]\right\}\left[1+c \ln \left(\frac{\tau_{2}}{\tau_{1}}\right)\right]^{-1} .
$$

Here $E_{1}(z)=\int_{z}^{\infty} \frac{e^{-t}}{t} d t, C$ represents the degree to which viscous effects are present, and $\tau_{1}$ and $\tau_{2}$ represent the fast and slow viscous time phenomena. We note that the internal strain variable formulation (2.1)-(2.3) is equivalent to the constitutive relationship proposed by Fung if one considers an approximation of the relaxation function $G$ by a sum of exponential terms. Various internal strain variable models are investigated in [2] and a good agreement is demonstrated between the two internal strain variable model $\left(\sigma=\varepsilon_{1}+\varepsilon_{2}\right)$ and undamped simulated data based on the Fung kernel $G$. We also remark that theoretical well-posedness results for the one strain variable model (which can be readily extended to treat finite multiple 
strain variables) are given in [14]. We further note that the derivations in [11], [12], and the references therein yield a molecular based foundation for internal variable models such as (2.2)-(2.3). These are generalizations of the linear stick-slip models of Doi and Edwards [20] and Johnson and Stacer [23].

As explained in the Introduction, here we are concerned with a generalization of the internal strain variable formulation. Fung's proposed model provides a continuous spectrum of relaxation times in contrast with the finite multiple internal strain model. In the latter, the material is assumed to have discrete relaxation rate constants, $\tau_{j}$, that correspond to a discrete hysteresis spectrum. As we have noted, Fung argues that this is incompatible with the observation that the hysteresis loop is independent of the strain rate within several decades of the rate variation for many biological soft tissues. Here we propose to realize a continuous spectrum of relaxation times in the form

$$
\sigma(t, x ; P)=C_{D} \dot{\varepsilon}(t)+\int_{0}^{t} G(t-s, P) \frac{d}{d s} \sigma_{e}\left(u_{x}(s)\right) d s
$$

with

$$
G(t, P)=\int_{\mathcal{T}} g(t, \tau) d P(\tau)
$$

where $\mathcal{T}=\left[\tau_{1}, \tau_{2}\right] \subset(0, \infty), P$ is a probability density function on $\mathcal{T}$, and $g$ is a continuous function of relaxation times $\tau$ on $\mathcal{T}$. We note that the case $g(t, \tau)=e^{-\frac{1}{\tau} t}$ corresponds to a continuum of internal strain variable models "weighted" by the probability density function $P$. This can be seen readily by applying the variation of constants formula in (2.2)-(2.3) (with $\left.C_{j}=1, j=1, \ldots, N\right)$

$$
\begin{aligned}
& \varepsilon_{j}\left(t ; \tau_{j}\right)=\int_{0}^{t} e^{-\frac{1}{\tau_{j}}(t-s)} \frac{d}{d s} \sigma_{e}\left(u_{x}(s)\right) d s \\
& \sigma(t, x ; P)=C_{D} \dot{u}_{x}(t)+\int_{\mathcal{T}} \int_{0}^{t} e^{-\frac{1}{\tau_{j}}(t-s)} \frac{d}{d s} \sigma_{e}\left(u_{x}(s)\right) d s d P(\tau),
\end{aligned}
$$

(and this can be generalized using a product measure on $\mathcal{T} \times \mathcal{C}$ to include a continuum of $C_{j}$-s). In the next section we present existence-uniqueness results for the generalized model with constitutive relationship (2.7)-(2.8).

\section{Existence and uniqueness of weak solutions}

Motivated by the weak or variational form of the model derived in the previous section we consider the abstract system

$$
\begin{aligned}
& u_{t t}-\frac{\partial}{\partial x} \sigma(t, x ; P)=F \text { in } V^{*} \\
& u(0, x)=u_{0} \in V=H^{1}\left(R_{1}, R_{2}\right) \\
& u_{t}(0, x)=u_{1} \in H=L^{2}\left(R_{1}, R_{2}\right)
\end{aligned}
$$


with boundary conditions

$$
\begin{aligned}
& \sigma\left(t, R_{1} ; P\right)=f(t) \\
& \sigma\left(t, R_{2} ; P\right)=0,
\end{aligned}
$$

where the shear stress $\sigma$ is given as

$$
\sigma(t, x ; P)=C_{D} \dot{u}_{x}(t, x)+\int_{0}^{t} G(t-s, P) \frac{d}{d s} \sigma_{e}\left(u_{x}(s, x)\right) d s,
$$

with

$$
G(t, P)=\int_{\mathcal{T}} g(t, \tau) d P(\tau)
$$

and $P$ is a probability density function on $\mathcal{T}$. We will denote the norm and inner product in $\mathrm{H}$ by $\|\cdot\|$ and $\langle\cdot, \cdot\rangle$, while all other norms and inner products will be appropriately labeled. We note that $\|v\|_{V}^{2}=\|v\|^{2}+\left\|v_{x}\right\|^{2}$, and that $V$ is compactly embedded in $H$ with $V \hookrightarrow H \hookrightarrow V^{*}$ forming a Gelfand triple [29].

We make the following assumptions

(A1) The forcing term $F$ satisfies $F \in L^{2}\left(0, T ; V^{*}\right)$.

(A2) The inner boundary condition satisfies $f \in L^{2}(0, T)$.

(A3) The elastic response function $\sigma_{e}$ satisfies the following local Lipschitz condition

$$
\left\|\sigma_{e}(u)-\sigma_{e}(v)\right\| \leq L_{B_{r}}\|u-v\|
$$

for some positive constant $L_{B_{r}}$ and for all $u, v \in B_{H}(0, r)$, the ball in $H$ centered at 0 of radius $r$.

(A4) There exist positive constants $C_{1}$ and $C_{2}$ such that

$$
\left\|\sigma_{e}(u)\right\| \leq C_{1}\|u\|+C_{2}
$$

for every $u \in H$.

We remark that these are the same assumptions as those of [14]. In addition, we make the following assumption on the relaxation function $G(t, P)=\int_{\mathcal{T}} g(t-s, \tau) d P(\tau)$.

(A5) The function $g$ is continuous in $\tau$ on $\mathcal{T}=\left[\tau_{1}, \tau_{2}\right] \subset(0, \infty), g$ is differentiable with respect to $t$ on $\mathbb{R}^{+}$, and there exist constants $C_{3}$ and $C_{4}$ such that $|g(t, \tau)| \leq C_{3}$ and $|\dot{g}(t, \tau)| \leq C_{4}$ for all $t \in \mathbb{R}^{+}$and all $\tau \in \mathcal{T}$.

We note that $g(t, \tau)=e^{-\frac{1}{\tau} t}$, which corresponds to a continuum of internal strain variable models, satisfies this latter condition.

We define weak solutions for the system (3.1)-(3.7) in the following way. 
Definition 3.1 Let $\mathcal{L}_{T}=\left\{w:[0, T] \rightarrow H, w \in C_{W}(0, T ; V) \cap L^{2}(0, T ; V)\right.$ and $w_{t} \in$ $\left.C_{W}(0, T ; H) \cap L^{2}(0, T ; V)\right\}$. We say that $u \in \mathcal{L}_{T}$ is a weak solution of the system (3.1)-(3.7) if it satisfies

$$
\begin{aligned}
& \int_{0}^{t}\left[-\left\langle u_{s}(s), \eta_{s}(s)\right\rangle+C_{D}\left\langle u_{s x}(s), \eta_{x}(s)\right\rangle+\left\langle\int_{0}^{s} G(s-\nu, P) \frac{d}{d s} \sigma_{e}\left(u_{x}(s)\right) d \nu, \eta_{x}(s)\right\rangle\right] d s \\
& +\left\langle u_{t}(t), \eta(t)\right\rangle-\left\langle u_{1}, \eta(0)\right\rangle=\int_{0}^{t}\left[\langle F(s), \eta(s)\rangle_{V^{*}, V}-f(s) \eta\left(s, R_{1}\right)\right] d s
\end{aligned}
$$

for any $t \in[0, T]$ and $\eta \in \mathcal{L}_{T}$ with initial conditions $u_{0} \in V$ and $u_{1} \in H$.

As in [14] we note that this notion of the weak solution for system (3.1)-(3.7) agrees with the usual one in that it yields $u_{t t} \in L^{2}\left(0, T ; V^{*}\right)$ with equation (3.1) holding in the sense of $L^{2}\left(0, T ; V^{*}\right)$.

Our first result is that the system (3.1)-(3.7) has a unique weak solution.

Theorem 3.1 Under assumptions (A1)-(A5) the system (3.1)-(3.7) has a unique global weak solution on any finite interval $[0, T]$.

Proof: Our arguments to establish this statement essentially follow those of [14]. Thus we present only a short outline and point out the similarities between the one internal variable model of [14] and its generalization considered here. First we note that the constitutive relationship $\sigma=C_{D} \dot{u}_{x}+\varepsilon_{1}$ that was used in [14] leads to

$$
\begin{aligned}
& \sigma(t)=C_{D} \dot{u}_{x}(t)+\int_{0}^{t} e^{-\frac{1}{\tau}(t-s)} \frac{d}{d s} \sigma_{e}\left(u_{x}(s)\right) d s \\
& =C_{D} \dot{u}_{x}(t)+\sigma_{e}\left(u_{x}(t)\right)-e^{-\frac{1}{\tau} t} \sigma_{e}\left(u_{0 x}\right)-\int_{0}^{t} \frac{d}{d s}\left(e^{-\frac{1}{\tau}(t-s)}\right) \sigma_{e}\left(u_{x}(s)\right) d s,
\end{aligned}
$$

while here we propose

$$
\begin{aligned}
& \sigma(t)=C_{D} \dot{u}_{x}(t)+\int_{0}^{t} G(t-s, P) \frac{d}{d s} \sigma_{e}\left(u_{x}(s)\right) d s \\
& =C_{D} \dot{u}_{x}(t)+G(0, P) \sigma_{e}\left(u_{x}(t)\right)-G(t, P) \sigma_{e}\left(u_{0 x}\right)+\int_{0}^{t} \dot{G}(t-s, P) \sigma_{e}\left(u_{x}(s)\right) d s,
\end{aligned}
$$

with

$$
G(t, P)=\int_{\mathcal{T}} g(t, \tau) d P(\tau)
$$

Thus as long as $G(t, P)$ has the properties that were used in [14] in connection with the exponential kernel, we can essentially repeat all the arguments there and can arrive at the desired conclusion. We remark that assumption (A5) guarantees this analogy between the two problems. Hence we can proceed as in [14] and as a first step establish the local existence of weak solutions under the assumptions (A1)-(A3) and (A5). To this end we define the radial retraction $P_{1}$ from $H$ onto $B_{H}\left(u_{0 x}, 1\right)$ and let $\hat{\sigma}_{e}=\sigma_{e}\left(P_{1} u\right)$, for $u \in H$. We note that $\hat{\sigma}_{e}$ satisfies a global Lipschitz property

$$
\left\|\hat{\sigma}_{e}(u)-\hat{\sigma}_{e}(v)\right\| \leq L\|u-v\| \text { for all } u, v \in H,
$$


and a boundedness property

$$
\left\|\hat{\sigma}_{e}(u)\right\| \leq \tilde{C}_{1}\|u\|+\tilde{C}_{2}
$$

for all $u \in H$ with positive constants $\tilde{C}_{1}$ and $\tilde{C}_{2}$.

Next, we let $\left\{\psi_{i}\right\}_{i=1}^{\infty}$ be any linearly independent total subset of $\mathrm{V}$, and for any $m, m=$ $1, \ldots$, we form $V^{m}=\operatorname{span}\left\{\psi_{1}, \ldots, \psi_{m}\right\}$. We choose sequences $\left\{u_{0}^{m}\right\}$ and $\left\{u_{1}^{m}\right\}$ with $u_{i}^{m}$ in $V^{m}, i=0,1$, such that $u_{0}^{m} \rightarrow u_{0}$ in $V$ and $u_{1}^{m} \rightarrow u_{1}$ in $H$ as $m \rightarrow \infty$. We define the Galerkin approximation

$$
u^{m}(t)=\sum_{k=1}^{m} a_{k}^{m}(t) \psi_{k}
$$

as the unique solution of the m-dimensional integro-differential system

$$
\begin{aligned}
& \left\langle u_{t t}^{m}, \psi_{j}\right\rangle_{V^{*}, V}+C_{D}\left\langle u_{t x}^{m}, \psi_{j x}\right\rangle+\left\langle\int_{0}^{t} G(t-s, P) \frac{d}{d s} \hat{\sigma}_{e}\left(u_{x}^{m}(s)\right) d s, \psi_{j x}\right\rangle \\
& =\left\langle F(t), \psi_{j}\right\rangle_{V^{*}, V}-f(t) \psi_{j}\left(R_{1}\right)
\end{aligned}
$$

for $j=1, \ldots, m$ on the interval $[0, T]$, for some $T>0$. Now we can use the same techniques as in [14] to establish an a priori estimate

$$
\left\|u_{t}^{m}(t)\right\|^{2}+\left\|u_{x}^{m}(t)\right\|^{2}+C_{D} \int_{0}^{t}\left\|u_{s x}^{m}(s)\right\|^{2} d s \leq K, \quad t \in[0, T]
$$

where $K=K\left(u_{0}, u_{1}, f, F, C_{D}, T, \hat{\sigma}_{e}, g, P\right)$ and is independent of $m$. Thus we can conclude that there exists a function $u \in L^{2}(0, T ; V)$ such that

$$
u^{m} \rightarrow u \text { weakly in } L^{2}(0, T ; V)
$$

and

$$
u_{t}^{m} \rightarrow u_{t} \text { weakly in } L^{2}(0, T ; V) .
$$

The following additional convergences that play a crucial role in the proof can also be established by the same methods as those used in $[10,14]$ (Ascoli-Arzela Theorem, Aubin's lemma).

$(\mathrm{R} 1) u^{m}(t) \rightarrow u(t)$ weakly in $V$ uniformly in $t \in[0, T]$, i.e., $u^{m} \rightarrow u$ in $\left.C_{W}(0, T ; V)\right)$.

$(\mathrm{R} 2) u_{t}^{m}(t) \rightarrow u_{t}(t)$ weakly in $H$ uniformly in $t \in[0, T]$, i.e., $u_{t}^{m} \rightarrow u_{t}$ in $\left.C_{W}(0, T ; H)\right)$.

(R3) $u_{t}^{m} \rightarrow u_{t}$ in $L^{2}(0, T ; H)$.

(R4) There exists a function $h \in L^{2}(0, T ; H)$ such that

$$
\int_{0}^{t} G(t-s) \hat{\sigma}_{e}\left(u_{x}(s)\right) d s \rightarrow h
$$

weakly in $L^{2}(0, T ; H)$. 
We remark that these convergences also hold on any subinterval of $[0, T]$. Now we verify that $u$ is a weak solution of (3.1)-(3.7) with $\hat{\sigma}_{e}$ instead of $\sigma_{e}$. First we define the class of functions $\mathcal{B}_{M}=\left\{\eta \in \mathcal{L}_{T} \mid \eta(t)=\sum_{k=1}^{M} c_{k}(t) \psi_{k}, c_{k} \in C^{1}[0, T]\right\}$, and note that $\mathcal{B}=\cup_{M=1}^{\infty} \mathcal{B}_{M}$ is dense in $\mathcal{L}_{T}$. Now we multiply (3.8) by $c_{k}(t)$, sum from 1 to $\mathrm{M}$ and integrate over $(0, t)$ to obtain that for all $\eta \in \mathcal{B}_{M}, m \geq M$

$$
\begin{aligned}
& \int_{0}^{t}\left[\left\langle u_{s s}^{m}(s), \eta(s)\right\rangle_{V^{*}, V}+C_{D}\left\langle u_{s x}^{m}(s), \eta_{x}(s)\right\rangle+\left\langle\int_{0}^{s} G(s-\nu, P) \hat{\sigma}_{e}\left(u_{x}^{m}(\nu)\right) d \nu, \eta_{x}(s)\right\rangle\right] d s \\
& =\int_{0}^{t}\langle F(s), \eta(s)\rangle_{V^{*}, V}-f(s) \eta\left(s, R_{1}\right) d s .
\end{aligned}
$$

We integrate the first term by parts and then take the limit as $m \rightarrow \infty$ using the convergences (R1)-(R4) to arrive at

$$
\begin{aligned}
& \int_{0}^{t}\left[-\left\langle u_{s}(s), \eta_{s}(s)\right\rangle+C_{D}\left\langle u_{s x}(s), \eta_{x}(s)\right\rangle+\left\langle h(s), \eta_{x}(s)\right\rangle\right] d s \\
& +\left\langle u_{t}(t), \eta(t)\right\rangle-\left\langle u_{1}, \eta(0)\right\rangle \\
& =\int_{0}^{t}\langle F(s), \eta(s)\rangle_{V^{*}, V}-f(s) \eta\left(s, R_{1}\right) d s
\end{aligned}
$$

for all $\eta \in \mathcal{L}_{T}$. Thus we can claim that $u$ is a weak solution of (3.1)-(3.7) (with $\sigma_{e}$ replaced by $\hat{\sigma}_{e}$ ) if we show that

$$
\int_{0}^{t}\left\langle h(s), \eta_{x}(s)\right\rangle d s=\int_{0}^{t}\left\langle\int_{0}^{s} G(s-\nu, P) \hat{\sigma}_{e}\left(u_{x}(\nu)\right) d \nu, \eta_{x}(s)\right\rangle d s
$$

for all $\eta \in \mathcal{L}_{T}$. This is achieved by establishing the strong convergence $u_{x}^{m}(t) \rightarrow u_{x}(t)$ in $\mathrm{H}$ as $m \rightarrow \infty$, again using the same arguments as in [14]. Uniqueness of weak solutions is proved the standard way.

Now we claim that the original system (3.1)-(3.7) with $\sigma_{e}$ has a unique local weak solution. Since $u_{x}$ is continuous in $t\left([19]\right.$, p.555) it follows that there exists $t^{*}, 0<t^{*} \leq T$ such that

$$
\left\|u_{x}(t)-u_{0 x}\right\| \leq 1
$$

for all $t \in\left[0, t^{*}\right]$. Therefore

$$
\sigma_{e}\left(u_{x}(t)\right)=\hat{\sigma}_{e}\left(u_{x}(t)\right), \quad t \in\left[0, t^{*}\right]
$$

i.e., $u$ is a weak solution of $(3.1)-(3.7)$ on $\left[0, t^{*}\right]$.

Finally, we can conclude the proof of Theorem 3.1 by showing that this local solution actually exists on any arbitrary interval $[0, T]$. This is accomplished by utilizing the boundedness assumption (A4) to obtain an a priori estimate similar to (3.9) for Galerkin approximations involving $\sigma_{e}$ instead of $\hat{\sigma}_{e}$, and then argue that $u_{x}(t)$ is pointwise bounded. The local Lipschitz condition (A3) can then be applied and similar steps to those above can be repeated to show that $u$ is the unique weak solution of (3.1)-(3.7). 


\section{Continuous dependence}

In this section we show that the unique weak solution of system (3.1)-(3.7) depends continuously on the "distributed relaxation times". This result is important for our ultimate goal of identifying stenosis, i.e., in considerations involving the inverse problem. To this end, we first introduce a topology on the probability distribution functions (PDFs) $P \in \mathcal{P}\left(\left[\tau_{1}, \tau_{2}\right]\right.$ ), where $\mathcal{P}\left(\left[\tau_{1}, \tau_{2}\right]\right)$ is the set of all PDFs on the Borel subsets of $\left[\tau_{1}, \tau_{2}\right]$. A convenient topology that will also be useful in treating the general inverse problem is given by the Prohorov metric $[3,4,15]$. For the sake of completeness we give the basic definitions and results concerning this metric here.

Suppose that $(Q, d)$ is a complete metric space, and for any closed subset $F \subset Q$ and $\epsilon>0$ define the set

$$
F^{\epsilon}=\{q \in Q: d(\tilde{q}, q)<\epsilon, \tilde{q} \in F\} .
$$

Then the Prohorov metric $\rho: \mathcal{P}(Q) \times \mathcal{P}(Q) \rightarrow \mathbb{R}^{+}$is defined by

$$
\rho\left(P_{1}, P_{2}\right) \equiv \inf \left\{\epsilon>0: P_{1}[F] \leq P_{2}\left[F^{\epsilon}\right]+\epsilon, F \text { closed, } F \subset Q\right\}
$$

It is well-known that

(1) $(\mathcal{P}(Q), \rho)$ is a complete metric space, and

(2) if $Q$ is compact then $(\mathcal{P}(Q), \rho)$ is a compact metric space.

The crucial properties of this metric in connection with our continuous dependence and inverse problems are summarized in the following theorem $([15])$.

Theorem 4.1 Given $P_{k}, P \in \mathcal{P}(Q)$, the following convergence statements are equivalent

(a) $\rho\left(P_{k}, P\right) \rightarrow 0$;

(b) $\int_{Q} f d P_{k}(q) \rightarrow \int_{Q} f d P(q)$ for all bounded, uniformly continuous functions $f: Q \rightarrow \mathbb{R}$;

(c) $P_{k}[A] \rightarrow P[A]$ for all Borel sets $A \subset Q$ with $P[\partial A]=0$;

We can now proceed to show that the weak solution of system (3.1)-(3.7) depends continuously on the probability distribution function $P$ in the Prohorov metric topology.

Theorem 4.2 Assume that assumptions (A1)-(A5) hold. If $P_{k} \rightarrow P$ in the Prohorov metric $\rho$, then $u_{k} \rightarrow u$ in $L^{2}(0, T ; V)$ and $u_{k t} \rightarrow u_{t}$ in $L^{2}(0, T ; V)$, where $u_{k}$ are the weak solutions corresponding to probability density functions $P_{k}$ and $u$ is the weak solution corresponding to $P$.

Proof: We first note that if $P_{k} \rightarrow P$ and $G$ is defined as $G(t, P)=\int_{\mathcal{T}} g(t, \tau) d P(\tau)$, where $g$ satisfies (A5), then by Theorem 4.1. we have that

$$
\begin{aligned}
& G\left(t, P_{k}\right) \rightarrow G(t, P) \text { for every } t \geq 0 \\
& \dot{G}\left(t, P_{k}\right) \rightarrow \dot{G}(t, P) \text { for every } t \geq 0
\end{aligned}
$$


We also remark that (A5) implies that

$$
\begin{aligned}
& \left|G\left(t, P_{k}\right)\right| \leq C_{3}, \text { for every } k, k=1, \ldots \\
& \left|\dot{G}\left(t, P_{k}\right)\right| \leq C_{4}, \text { for every } k, k=1, \ldots
\end{aligned}
$$

Now we have that

$$
\sigma(t, x ; P)=C_{D} \dot{\varepsilon}+\int_{0}^{t} G(t-s) \frac{d}{d s}\left(\sigma_{e}\left(u_{x}(s)\right)\right) d s=C_{D} \dot{\varepsilon}+E_{1}(t, \varepsilon ; P),
$$

where $\varepsilon=u_{x}$, and by the above remarks $E_{1}\left(t, \varepsilon ; P_{k}\right) \rightarrow E_{1}(t, \varepsilon, P)$ for a fixed $\varepsilon$, and any $t \geq 0$ whenever $P_{k} \rightarrow P$ in the Prohorov metric. Let $u_{k}^{m}, u^{m}$ denote the Galerkin approximations corresponding to the weak solutions $u_{k}$ and $u$ of (3.1)-(3.7) with respective PDFs $P_{k}$ and $P$. We note that the initial conditions do not depend on the PDFs $P_{k}$ or $P$. Thus we have

$$
\left\langle u_{t t}^{m}, \psi_{j}\right\rangle_{V^{*}, V}+C_{D}\left\langle u_{t x}^{m}, \psi_{j x}\right\rangle+\left\langle E_{1}\left(u_{x}^{m} ; P\right), \psi_{j x}\right\rangle=\left\langle F(t), \psi_{j}\right\rangle_{V^{*}, V}-f(t) \psi_{j}\left(R_{1}\right)
$$

and

$$
\left\langle u_{k t t}^{m}, \psi_{j}\right\rangle_{V^{*}, V}+C_{D}\left\langle u_{k t x}^{m}, \psi_{j x}\right\rangle+\left\langle E_{1}\left(u_{k x}^{m} ; P\right), \psi_{j x}\right\rangle=\left\langle F(t), \psi_{j}\right\rangle_{V^{*}, V}-f(t) \psi_{j}\left(R_{1}\right),
$$

which yields

$$
\left\langle u_{k t t}^{m}-u_{t t}^{m}, \psi_{j}\right\rangle_{V^{*}, V}+C_{D}\left\langle u_{k t x}^{m}-u_{t x}^{m}, \psi_{j x}\right\rangle+\left\langle E_{1}\left(u_{k x}^{m} ; P\right)-E_{1}\left(u_{x}^{m} ; P\right), \psi_{j x}\right\rangle=0
$$

Let $\Delta E_{1}^{m}=E_{1}\left(u_{k x}^{m} ; P\right)-E_{1}\left(u_{x}^{m} ; P\right)$. We remark that by the above observation on the boundedness of $G$ and $\dot{G}$ we have that the same a priori estimates can be shown to be valid for both the Galerkin approximations $\left\{u_{k}^{m}\right\}$ and $\left\{u^{m}\right\}$, i.e., there exists a constant $K$ independent of $m$ and $k$ such that

$$
\begin{aligned}
& \left\|u_{k t}^{m}(t)\right\|^{2}+\left\|u_{k x}^{m}(t)\right\|^{2}+C_{D} \int_{0}^{t}\left\|u_{k s x}^{m}(s)\right\|^{2} d s \leq K \\
& \left\|u_{t}^{m}(t)\right\|^{2}+\left\|u_{x}^{m}(t)\right\|^{2}+C_{D} \int_{0}^{t}\left\|u_{s x}^{m}(s)\right\|^{2} d s \leq K,
\end{aligned}
$$

for all $t \in[0, T], \quad m=1, \ldots$ Since these estimates guarantee the pointwise boundedness of $\left\|u_{x}^{m}\right\|$ and $\left\|u_{x}\right\|$ (by the weak lower semicontinuity of norms and the convergence $u_{x}^{m}(t) \rightarrow$ $u_{x}(t)$ in $\left.\mathrm{H}\right)$, we can utilize the local Lipschitz property (A3) of $\sigma_{e}$ in $B_{H}(0, \sqrt{K})$ together with the growth condition (A4) to obtain the following estimate for $\Delta E_{1}^{m}$

$$
\begin{aligned}
& \left\|\Delta E_{1}^{m}(t)\right\| \leq\left|G\left(0, P_{k}\right)\right| L_{\sqrt{K}}\left\|u_{k x}^{m}(t)-u_{x}^{m}(t)\right\|+\left|G\left(0, P_{k}\right)-G(0, P)\right|\left\{C_{1}\left\|u_{x}^{m}(t)\right\|+C_{2}\right\} \\
& +\left|G\left(t, P_{k}\right)\right| L_{\sqrt{K}}\left\|u_{k x}^{m}(0)-u_{x}^{m}(0)\right\|+\left|G\left(t, P_{k}\right)-G(t, P)\right|\left\{C_{1}\left\|u_{x}^{m}(0)\right\|+C_{2}\right\} \\
& +\int_{0}^{t}\left[\left|\dot{G}\left(t-s, P_{k}\right)\right| L_{\sqrt{K}}\left\|u_{k x}^{m}(s)-u_{x}^{m}(s)\right\|\right. \\
& \left.+\left|\dot{G}\left(t-s, P_{k}\right)-\dot{G}(t-s, P)\right|\left\{C_{1} \| u_{x}^{m}(s)+C_{2}\right\}\right] d s .
\end{aligned}
$$


Let $\Delta_{k}^{m}(t)=u_{k}^{m}(t)-u^{m}(t)$. The a priori estimate yields that there exist positive constants $M_{1}, M_{2}$ and $M_{3}$ such that

$$
\begin{aligned}
& \left\|\Delta E_{1}^{m}(t)\right\| \leq M_{1}\left\|\Delta_{k x}^{m}(t)\right\|+M_{3}\left|G\left(0, P_{k}\right)-G(0, P)\right|+M_{3}\left|G\left(t, P_{k}\right)-G(t, P)\right| \\
& \int_{0}^{t}\left[M_{2}\left\|\Delta_{k x}^{m}(s)\right\|+M_{3}\left|\dot{G}\left(t-s, P_{k}\right)-\dot{G}(t-s, P)\right|\right] d s,
\end{aligned}
$$

where we used the fact that $u_{k x}^{m}(0)-u_{x}^{m}(0)=0$. We now return to (4.5), multiply by $a_{j k t}^{m}(t)-a_{j t}^{m}(t)$ and sum over $j$ to obtain

$$
\frac{1}{2} \frac{d}{d t}\left\|\Delta_{k t}^{m}(t)\right\|^{2}+C_{D}\left\|\Delta_{k t x}^{m}(t)\right\|^{2}+\left\langle\Delta E_{1}^{m}(t), \Delta_{k t x}^{m}(t)\right\rangle=0 .
$$

We add $\left\langle\Delta_{k x}^{m}(t), \Delta_{k t x}^{m}(t)\right\rangle$ to both sides, and then using standard inequalities we arrive at

$$
\frac{1}{2} \frac{d}{d t}\left(\left\|\Delta_{k t}^{m}(t)\right\|^{2}+\left\|\Delta_{k x}^{m}(t)\right\|^{2}\right)+\frac{C_{D}}{2}\left\|\Delta_{k t x}^{m}(t)\right\|^{2} \leq \frac{2}{C_{D}}\left(\left\|\Delta E_{1}^{m}(t)\right\|^{2}+\left\|\Delta_{k x}^{m}(t)\right\|^{2}\right) .
$$

Let us introduce the notation

$$
\Delta G(t, k)=G\left(t, P_{k}\right)-G(t, P)
$$

and

$$
\Delta \dot{G}(t, k)=\dot{G}\left(t, P_{k}\right)-\dot{G}(t, P) .
$$

By the estimate (4.6) we have

$$
\begin{aligned}
& \left\|\Delta E_{1}^{m}(t)\right\|^{2} \leq M_{4}\left\{\left\|\Delta_{k x}^{m}(t)\right\|^{2}+|\Delta G(0, k)|^{2}+|\Delta G(t, k)|^{2}+t \int_{0}^{t}\left\|\Delta_{k x}^{m}(s)\right\|^{2} d s\right. \\
& \left.+\int_{0}^{t}|\Delta \dot{G}(t-s, k)|^{2} d s\right\} .
\end{aligned}
$$

Next we integrate (4.7) from 0 to $t$ and obtain after using standard methods and the estimate (4.8) that

$$
\left\|\Delta_{k t}^{m}(t)\right\|^{2}+\left\|\Delta_{k x}^{m}(t)\right\|^{2}+C_{D} \int_{0}^{t}\left\|\Delta_{k t x}^{m}(s)\right\|^{2} d s \leq K_{1} \int_{0}^{t}\left\|\Delta_{k x}^{m}(s)\right\|^{2} d s+\mathcal{J}_{k}(t),
$$

where $\mathcal{J}_{k}(t)$ is given by

$$
\mathcal{J}_{k}(t)=\frac{2 M_{2}}{C_{D}} \int_{0}^{t}\left\{|\Delta G(0, k)|^{2}+|\Delta G(s, k)|^{2}+\frac{t^{2}}{2}|\Delta \dot{G}(t-s, k)|^{2}\right\} d s .
$$

By (4.1)-(4.2) and (4.3)-(4.4) we can assert that

$$
\mathcal{J}_{k}(t) \rightarrow 0
$$

for $t \in[0, T]$ as $k \rightarrow \infty$. Application of Gronwall's lemma in (4.9) yields

$$
\left\|\Delta_{k t}^{m}(t)\right\|^{2}+\left\|\Delta_{k x}^{m}(t)\right\|^{2} \leq \mathcal{J}_{k}(t)+K_{1} \int_{0}^{t} \mathcal{J}_{k}(s) e^{K_{1}(t-s)} d s, \quad t \in[0, T] .
$$


By the weak lower semicontinuity of norms, the convergences (R1) and (R3) for a fixed $k$ and (4.10) we obtain from (4.11) that

$$
u_{k t}(t) \rightarrow u_{t}(t) \text { in } H \text { for each } t \in[0, T]
$$

and

$$
u_{k x}(t) \rightarrow u_{x}(t) \text { in } H \text { for each } t \in[0, T]
$$

We note that since

$$
\left\|\Delta_{k}^{m}(t)-\Delta_{k}^{m}(0)\right\| \leq \int_{0}^{t}\left\|\Delta_{k t}^{m}\right\| d s
$$

implies

$$
\left\|\Delta_{k}^{m}(t) \leq\right\| \Delta_{k}^{m}(0)\left\|+\int_{0}^{t}\right\| \Delta_{k t}^{m} \| d s
$$

which tends to 0 as $k \rightarrow \infty$, we also have that

$$
u_{k}(t) \rightarrow u(t) \text { in } H \text { for each } t \in[0, T]
$$

Additionally, we can show that $u_{k t x} \rightarrow u_{t x}$ in $L^{2}(0, T ; H)$ by using the estimate (4.9). Thus we can conclude that $u_{k} \rightarrow u$ in $L^{2}(0, T ; V)$ and $u_{k t} \rightarrow u_{t}$ in $L^{2}(0, T ; V)$, which establishes Theorem 4.2. We note that the proof actually shows that the map $P \rightarrow u(t ; P)$ is continuous from $\mathcal{P}(Q)$ to $V$ for each $t \in[0, T]$.

\section{The inverse problem}

Since the final goal of the original investigation was the detection of stenosis in arteries, in this section we turn to the study of the inverse problem. In particular, we consider whether it is possible to determine mechanical properties of the tissue (i.e., the distribution of relaxation times) by measuring shear wave propagation at the surface of the chest wall. Thus we attempt to estimate $P \in \mathcal{P}(Q)$, (where $\mathcal{P}(Q)$ is the set of all probability density functions over $\mathrm{Q}$ ), given, e.g., shear displacement data $\hat{d}_{i}, \quad i=1 \ldots, n$, by minimizing the cost functional

$$
J(P)=\sum_{i=1}^{n}\left|u\left(t_{i}, R_{2} ; P\right)-\hat{d}_{i}\right|^{2}
$$

over all $P \in \mathcal{P}(Q)$. We assume that $Q$ is compact. Our continuous dependence result in the previous section (i.e., $P \rightarrow u(t ; P)$ is continuous from $\mathcal{P}(Q)$ to $\mathrm{V}, Q=\mathcal{T}$, compact) implies that the map $P \rightarrow J(P)$ is continuous from $\mathcal{P}(Q)$ to $\mathbb{R}$. Since the compactness of $Q$ guarantees that of $\mathcal{P}(Q)$, we have that minimizer must exist, i.e., the inverse problem has a solution. The framework developed in [3] also provides a way to approximate solutions of the inverse problem that is useful in actual computations. It is shown that an arbitrary element 
of $\mathcal{P}(Q)$ can be approximated by a finite linear combination of Dirac measures. To be more precise, let $Q_{M}=\left\{q_{j}^{M}\right\}_{j=1}^{M}, M=1,2, \ldots, \infty$, be subsets of $Q$ chosen so that $Q_{d}=\cup_{M=1}^{\infty} Q_{M}$ is dense in $Q$. Next define

$$
\mathcal{P}^{M}(Q)=\left\{P \in \mathcal{P}(Q) \mid P=\sum_{j=1}^{M} p_{j} \delta_{q_{j}^{M}}, q_{j}^{M} \in Q_{M}, p_{j} \text { rational, } \sum p_{j}=1\right\},
$$

where $\delta_{q}$ is the Dirac measure with atom at $q$. Then in [3] it is shown that any element of $\mathcal{P}(Q)$ can be approximated arbitrarily closely (in the Prohorov metric) by elements in $\mathcal{P}^{M}(Q)$ for $M$ sufficiently large. That is, $\cup_{M=1}^{\infty} \mathcal{P}^{M}(Q)$ is dense in $\mathcal{P}(Q)$ in the Prohorov metric. In terms of our problem of shear wave propagation this means that we can approximate with a model involving a finite number of internal strain variables in the numerical computations. The results of such an attempt are detailed in Section 6.

However, in some problems it is natural to minimize $\mathrm{J}(\mathrm{P})$ over a subset of $\mathcal{P}(Q)$ consisting of only absolutely continuous probability distributions, i.e., those that have 'continuous' densities with $\operatorname{Prob}[a, b]=\int_{a}^{b} f(x) d x$. For example, as we mentioned before, Fung argues against a finite spectrum of relaxation times (which results if one uses an approximation with finite linear combinations of Dirac measures) and suggests a continuous distribution of relaxation times. In this context it might be more desirable to minimize $J(F)$ over some $\mathcal{P}_{\mathcal{F}}(Q)=\left\{F \in \mathcal{P}(Q) \mid F=\int f, f \in \mathcal{F}\right\}$, where $\mathcal{F}$ is a given set of functions, and this motivates the development of new theoretical and approximation results. We remark that a crucial property in the Banks-Bihari [3] framework is that the compactness of Q implies compactness of the space $(\mathcal{P}(Q, \rho)$, where $\rho$ denotes the Prohorov metric. Thus we look for some type of compactness condition imposed on the set of functions $\mathcal{F}$ that leads to the compactness of $\mathcal{P}_{\mathcal{F}}(Q)$ in the $\rho$ metric. To this end we prove the following result.

Theorem 5.1 Let $\mathcal{F}$ be a weakly compact subset of $L^{2}(Q)$, with $Q$ compact, and let $\mathcal{P}_{\mathcal{F}}(Q)$ be the family of probability distribution functions on $Q$ generated by $\mathcal{F}$ as a set of densities

$$
\mathcal{P}_{\mathcal{F}}(Q)=\left\{F \in \mathcal{P}(Q) \mid F^{\prime}=f, f \in \mathcal{F}\right\}
$$

Then $\mathcal{P}_{\mathcal{F}}(Q)$ is a $\rho$ compact subset of $(\mathcal{P}(Q), \rho)$ where $\rho$ is the Prohorov metric on the set $\mathcal{P}(Q)$ of all probability density functions on $Q$.

Proof: Let $\left\{F_{n}\right\} \in \mathcal{P}_{\mathcal{F}}(Q)$, where $F_{n}=\int f_{n} d s, \quad f_{n} \in \mathcal{F}$. Since weak compactness of $\mathcal{F}$ implies that $\mathcal{F}$ is norm bounded we have that $\left\{f_{n}\right\} \subset \mathcal{F}$ is weakly sequentially compact, i.e., there exists an $f \in \mathcal{F}$ and a subsequence $\left\{f_{n_{k}}\right\}$ of $\left\{f_{n}\right\}$ such that $f_{n_{k}} \rightarrow f$ with $\left\|f_{n_{k}}\right\|_{L^{2}} \leq M$. Then for any continuous function $g \in C(Q)$ we have

$$
\int_{Q} g f_{n_{k}} d x \rightarrow \int_{Q} g f d x
$$

which in turn implies that $F_{n_{k}} \rightarrow F=\int f d s$ in the $\rho$-metric. Thus $\mathcal{P}_{\mathcal{F}}(Q)$ is sequentially compact. Next we establish that this set is also closed in the $\rho$-metric. Let $\left\{F_{n}\right\} \subset \mathcal{P}_{\mathcal{F}}(Q)$ 
be such that $F_{n} \rightarrow F \in \mathcal{P}(Q)$ in the $\rho$-metric. We must argue that $F \in \mathcal{P}_{\mathcal{F}}(Q)$. Since $F_{n}=\int f_{n} d s$, we have that

$$
\int_{Q} g f_{n} d x \rightarrow \int_{Q} g d F
$$

for every $g \in C(Q)$. Since $\left\{f_{n}\right\} \subset \mathcal{F}$ we can assert as above that there exists $\left\{f_{n_{k}}\right\}$ and $f \in \mathcal{F}$ such that

$$
\int_{Q} g f_{n_{k}} d x \rightarrow \int_{Q} g f d x
$$

for all $g \in C(Q)$, which implies that

$$
\int_{Q} h f_{n_{k}} d x \rightarrow \int_{Q} h f d x
$$

for every $h \in L^{2}(Q)$, since $\left\{f_{n_{k}}\right\}$ is bounded in $L^{2}(Q)$ and $C(Q)$ is dense in $L^{2}(Q)$. Now by $(5.2)$

$$
\int_{Q} \tilde{g} d F=\int_{Q} \tilde{g} f d x \text { for all } \tilde{g} \in L^{2}(Q),
$$

i.e., $F$ is absolutely continuous with $F^{\prime}=f$, which implies that $F \in \mathcal{F}$. Thus we can conclude that $\mathcal{P}_{\mathcal{F}}(Q)$ is compact in $(\mathcal{P}(Q), \rho)$.

This result, combined with the continuous dependence statement above, guarantees that the inverse problem is solvable, i.e., a minimizer of the cost functional $J(P)$ exists over the set $\mathcal{P}_{\mathcal{F}}(Q) \subset \mathcal{P}(Q)$. We note, that although the previous computational framework utilizing Dirac measures is valid here, it may be desirable to develop 'smoother' approximations to elements of $\mathcal{P}_{\mathcal{F}}(Q)$. Namely, suppose that $f \in \mathcal{F}$ and $F \in \mathcal{P}(Q)$ with $F=\int f$. Since $\mathcal{F} \subset L^{2}(Q)$, we can formulate a piecewise linear spline approximation to $f$, i.e., let

$$
f^{N}(x)=\sum_{j=0}^{N} b_{j}^{N} \ell_{j}^{N}(x),
$$

(where the $\ell_{j}$-s are the usual piecewise linear splines) such that $f^{N} \rightarrow f$ in $L^{2}(Q)$, and the $b_{j}^{N}$-s are rational numbers. This implies that

$$
\int_{Q} g f^{N} d x \rightarrow \int_{Q} g f d x
$$

for all $g \in L^{2}(Q)$, hence for all $g \in C(Q)$, which yields

$$
\rho\left(f^{N}, f\right) \rightarrow 0 .
$$

Let $\mathcal{F}^{N}=\left\{h \in L^{2}(Q) \mid h(x)=\sum_{j=0}^{N} b_{j}^{N} \ell_{j}^{N}(x)\right\}$, where the $b_{j}^{N}$-s are rational. Thus we can conclude that the set

$$
\tilde{\mathcal{P}}(Q)=\left\{F \in \mathcal{P}(Q) \mid F=\int f, f \in \cup_{1}^{\infty} \mathcal{F}^{N}\right\}
$$

is dense in $\mathcal{P}_{\mathcal{F}}(Q)$ in the $\rho$-metric. Hence piecewise linear splines provide an alternative way to approximate elements of $\mathcal{P}_{\mathcal{F}}(Q)$ in our computational work. (Recall that elements of $\cup \mathcal{P}^{M}(Q)$, which is a dense subset of $\mathcal{P}(Q)$, can also be used to approximate elements in $\mathcal{P}_{\mathcal{F}}(Q)$ even though the elements of $\mathcal{P}^{M}(Q)$ are not in $\mathcal{P}_{\mathcal{F}}(Q)$.) 


\section{A computational example}
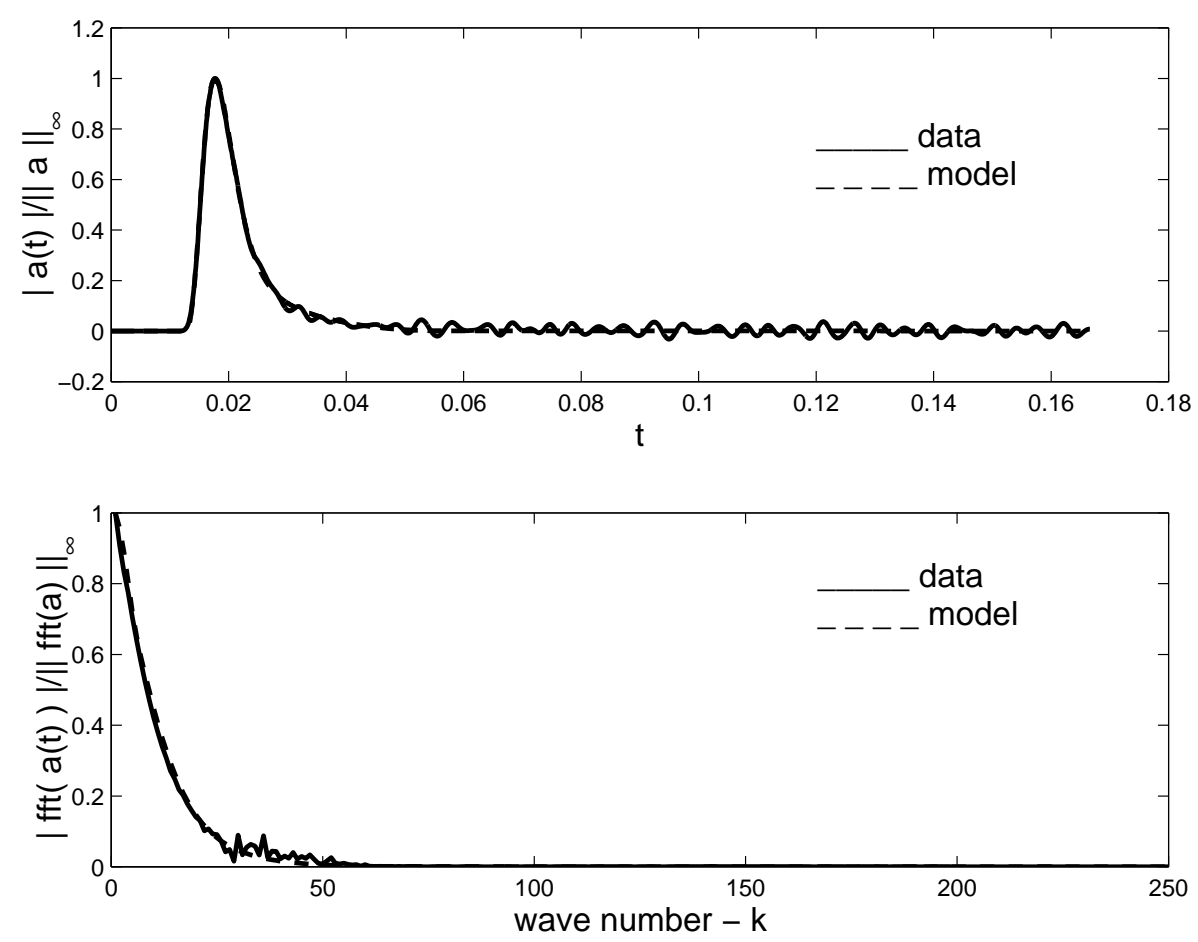

Figure 2: Comparison of the optimized model normalized acceleration with two internal variables (dashed line) and Verburg acceleration data (solid line)

In this section we present an inverse problem example to illustrate the computational aspects of the theory developed above. The particular example presented is part of the computational effort reported in [2]. "Data" was simulated using the experimental frequencies of Verburg [27] for a medium with mechanical properties similar to the lung tissue located between the heart valves and the chest wall. We shall refer to this as the Verburg data; details on precisely how this data is generated can be found in [2] but we note it contains no damping or dissipation. It was used as observations for shear acceleration $\hat{a}_{i}$ at $R_{2}$ in a least squares formulation similar to (5.1). The model chosen was of the form (2.1)-(2.3) with $C_{D}=0$ and $\sigma_{e}$ given by (2.4) with $\gamma=-\beta=-1.0$ taken fixed. In the optimization problem, the class of measures was restricted to the set

$$
\mathcal{P}^{2}(Q)=\left\{P \in \mathcal{P}(Q) \mid P(\tau)=\frac{1}{2} \delta_{\tau_{1}}(\tau)+\frac{1}{2} \delta_{\tau_{2}}(\tau)\right\}
$$

where in this case $Q$ was taken as a Cartesian product of compact intervals of positive $\tau$ values, i.e., $Q=\left[\tau_{1}^{-}, \tau_{1}^{+}\right] \times\left[\tau_{2}^{-}, \tau_{2}^{+}\right]$. In the example we also estimated $C_{1}, C_{2}$ in $(2.2)$ as well as $\alpha$ in (2.4). Thus the search was carried out over five Euclidean parameters. The best fit parameters found were $C_{1}^{*}=44.78, C_{2}^{*}=96.023, \tau_{1}^{*}=.000416, \tau_{2}^{*}=.0038, \alpha^{*}=.24$. The 
model simulation with these optimal parameters is compared to the Verburg data in Figure 2 where comparison plots are given in both the time domain and the frequency domain.

We note that one could also use elements of $\tilde{\mathcal{P}}(Q)$ of $(5.3)$ in place of the elements from $\mathcal{P}^{M}(Q)$ in these problems. While such an approximation would yield a system with a continuous spectrum of relaxation times, the computational efforts in this class of problems promises to require somewhat more substantial efforts than use of the elements in $\mathcal{P}^{M}(Q)$. Since the elements in $\mathcal{P}^{2}(Q)$ performed quite well with the Verburg data, we did not pursue piecewise linear spline approximations for these problems. The authors of [2] did consider certain parameterized nonlinear versions of $(2.2)$, i.e., the $\frac{1}{\tau_{j}} \varepsilon_{j}$ term is replaced by a parameterized nonlinearity $g\left(\varepsilon_{i n t}, \bar{b}\right)$ consisting of piecewise linear splines in the internal strain, but they noted no improved performance over that when using the $\mathcal{P}^{M}(Q)$ approximate systems with the Verburg data. We note here that these nonlinear systems considered in [2], which can be written

$$
\frac{d \varepsilon_{\text {int }}}{d t}+g\left(\varepsilon_{\text {int }} ; \bar{b}\right)=C \frac{d}{d t} \sigma_{e}\left(u_{x}(t)\right)
$$

can also be readily treated by the probabilistic framework developed here. In this case, one can take measures over the parameters $\bar{b}$ and $C$, where $\varepsilon_{\text {int }}(t)=\varepsilon_{\text {int }}(t ; \bar{b}, C)$, instead of over the parameters $\tau$ and $C$.

\section{Concluding remarks}

In the above discussions we have presented a theoretical framework based on a probabilistic multiscale formulation for molecular based hysteresis in tissue-like materials. These efforts were motivated by and formulated in the context of shear propagation in which reptation for long strand molecules plays a fundamental role and where the molecular dynamic parameters vary across populations of molecules in an unknown, probabilistic manner. We developed an inverse problem approach for measure dependent dynamical systems (in this case, partial differential equations for shear displacements) by making use of the Prohorov metric topology on measures. The formulation offers a computationally tractable alternative to the wellknown Fung kernel approach for viscoelastic biomaterials.

While the motivating applications are important in their own right, we are confident that the underlying ideas have potential for a much wider class of applications. In addition to the areas mentioned in the Introduction, we feel that such an approach will eventually prove relevant in diverse areas such as electromagnetic polarization and conductivity in heterogeneous materials (see [9],[12] and the references cited therein), industrial polymeric melts $([16],[17],[18],[24])$, as well as in other biological applications [4] (e.g., disease pathogenesis, epidemiology, ecological migrations, and genomic to system response modelsbioinformatics!). The fact that the theoretical formulation leads readily to both computationally and theoretically sound approximations significantly enhances the attractiveness of this approach. 


\section{Acknowledgments}

This research was supported in part by the U.S. Air Force Office of Scientific Research under grants AFOSR F49620-01-1-0026 (HTB) and AFOSR F49620-03-1-0185 (GAP), in part by the Joint DMS/NIGMS Initiative to Support Research in the Area of Mathematical Biology under grant 1R01GM67299-01, and was facilitated through visits of the authors to the Statistical and Applied Mathematical Sciences Institute, (SAMSI), which is funded by NSF under grant DMS-0112069. The authors are grateful to Dr. Shannon Wynne for helpful discussions on the computational examples of [2].

\section{References}

[1] H.T. BANKS, Some remarks on estimation techniques for size-structured population models, in Frontiers in Mathematical Biology, (S. Levin, ed.), Lecture Notes in Biomathematics, Vol. 100, Springer Verlag, Berlin, 1994, pp. 609-623.

[2] H.T. Banks, J.H. Barnes, A. Eberhardt, H. Tran and S. Wynne, Modeling and computation of propagating waves from coronary stenosis, Comput. Appl. Math., 21 (2002), pp. 767-788.

[3] H.T. BANKS AND K.L. BIHARI, Modeling and estimating uncertainty in parameter estimation, Inverse Problems, 17 (2001), pp. 95-111.

[4] H.T. Banks, D. Bortz, G.A. Pinter And L.K. Potter, Modeling and imaging techniques with potential for application in bioterrorism, Chapter 6 in Bioterrorism: Mathematical Modeling Applications in Homeland Security, (H.T. Banks and C. Castillo-Chavez, eds.), Frontiers in Applied Mathematics, SIAM, Philadelphia, 2003, pp. 129-154.

[5] H.T. Banks, L.W. Botsford, F. Kappel and C. Wang, Modeling and estimation in size structured population models, Proc. 2nd Course on Math. Ecology, (Trieste, December, 1986), World Scientific Press, Singapore, 1988, pp. 521-541.

[6] H.T. BAnks And B.G. FitzPatrick, Estimation of growth rate distributions in sizestructured population models, Quart. Appl. Math., 49 (1991), pp. 215-235.

[7] H.T. Banks, B.G. FitzPatrick, L.K. Potter And Y. Zhang, Estimation of probability distributions for individual parameters using aggregate population observations, in Stochastic Analysis, Control, Optimization and Applications, (W.McEneaney, G. Yin, and Q. Zhang, eds.), Birkhäuser, Basel, 1998, pp. 353-371.

[8] H.T. Banks, B.G. FitzPatrick And Y. Zhang, Estimation of distributed individual rates from aggregate population data, in Differential Equations and Applications to Biology and to Industry, (M. Martelli, et. al., eds.), World Scientific Press, Singapore, 1996, pp. 13-22. 
[9] H.T. Banks And N.L. Gibson, Well-posedness in Maxwell systems with distributions of polarization relaxation parameters, CRSC-TR04-01, NCSU, January, 2004; Applied Math. Letters, submitted.

[10] H.T. Banks, D.S. Gilliam And V.I. Shubov, Global solvability for damped abstract nonlinear hyperbolic systems, Differential and Integral Equations, 10 (1997), pp. 309332.

[11] H.T. Banks, N.G. Medhin And G.A. Pinter, Multiscale considerations in modeling of nonlinear elastomers, CRSC-TR03-42, NCSU, October, 2003; J. Comp. Meth. Sci. and Engr., submitted.

[12] H.T. Banks, N.G. Medhin And G.A. Pinter, Nonlinear reptation in molecular based hysteresis models for polymers, CRSC-TR03-45, NCSU, December, 2003; Quart. Appl. Math., submitted.

[13] H.T. Banks And G.A. Pinter, Damping: hysteretic damping and models, CRSCTR99-36, NCSU, December, 1999; in Encyclopedia of Vibration ( S.G. Braun, D. Ewins and S. Rao, eds.), Academic Press, London, 2001, pp. 658-664.

[14] H.T. Banks, H. Tran And S. Wynne, A well-posedness result for a shear wave propagation model, Intl. Series Num. Math., Vol.143, Birkhauser Verlag, Basel, 2002, pp. 25-40.

[15] P. Billingsley, Convergence of Probability Measures, Wiley, New York, 1968.

[16] G. Bishko, T.C.B. McLeish, O.G. Harlen and R.G. Larson, Theoretical molecular rheology of branched polymers in simple and complex flows: The pom-pom model, Phys. Rev. Lett., 79 (1997), pp. 2352-2355.

[17] R. Blackwell, O.G. Harlen and T.C.B. McLeish, Theoretical linear and nonlinear rheology of symmetric treelike polymer melts, Macromolecules 34 (2001), pp. 25792596.

[18] R. Blackwell, T.C.B. McLeish and O.G. Harlen, Molecular drag-strain coupling in branched polymer melts, J. Rheology 44 (2000), pp. 121-136.

[19] R. Dautray And J.L. Lions, Mathematical Analysis and Numerical Methods for Science and Technology, Vol.5, Springer-Verlag, New York, 2000.

[20] M. Doi And M. Edwards, The Theory of Polymer Dynamics, Oxford, New York, 1986.

[21] J.D. Ferry, E.R. Fitzgerald, L.D. Grandine And M.L. Williams, Temperature dependence of dynamic properties of elastomers: relaxation distributions, Ind. Engr. Chem., 44 (1952), pp. 703-706. 
[22] Y.C. Fung, Biomechanics: Mechanical Properties of Living Tissues, Springer-Verlag, New York, NY, 1993.

[23] A.R. Johnson and R.G. StaceR, Rubber viscoelasticity using the physically constrained systems' stretches as internal variables, Rubber Chemistry Technology, 66 (1993), pp. 567-577.

[24] T.C.B. McLeish and R.G. Larson, Molecular constitutive equations for a class of branched polymers: The pom-pom polymer, J. Rheology 42 (1998), pp. 81-110.

[25] F. Schwarzl and A.J. Staverman, Higher approximation methods for the relaxation spectrum from static and dynamic measurements of viscoelastic materials, Appl. Sci. Res., A4 (1953), pp. 127-141.

[26] D. Ter HaAr, A phenomenological theory of viscoelastic behavior, Physica, 16 (1950), pp. 839-850.

[27] J. Verburg, Transmission of vibrations of the heart to the chestwall, Adv. Cardiovasc. Phys., 5 (1983), pp. 84-103.

[28] M.L. Williams And J.D. Ferry, Second approximation calculations of mechanical and electrical relaxation and retardation distributions, J. Poly. Sci., 11 (1953), pp. 169175 .

[29] J. Wloka, Partial Differential Equations, Cambridge Univ. Press., Cambridge, UK, 1987. 